\title{
Commentary
}

See article on page 700

\section{Homing-in on the origin of biliary steroids}

The raison d'être of bile is to provide a conduit to eliminate excess cholesterol from the organism. ${ }^{1}$ Biliary secretion accomplishes this function in two ways. The liver secretes unesterified (free) cholesterol molecules into bile and also converts cholesterol molecules into bile acids, which are secreted as water soluble anions (bile salts). Bile salt synthesis from cholesterol compensates for obligatory faecal loss (as acidic sterols) during enterohepatic cycling, and this feedback control is regulated by the efficiency of bile salt return from the intestine. In a healthy human of ideal body weight, de novo synthesis amounts to about $250 \mathrm{mg}$ a day and the bile salt pool, which approximates $3 \mathrm{~g}$, cycles 8 to 12 times a day; therefore, the contribution of bile salt synthesis to biliary secretion is approximately $1 \%$. In contrast to the efficiency of the enterohepatic circulation, 50\% of the $1000 \mathrm{mg}$ of cholesterol secreted daily into bile is lost in faeces as bacterial metabolic products (neutral sterols) in faeces, thereby exceeding the loss of cholesterol as acidic sterols by a factor of 2 to 1 .

Knowledge of the precursor sources and disposition of biliary cholesterol and bile salts is important for its own sake and to provide targets for new drugs because of the high prevalence rates of atherosclerosis and cholesterol gallstones in Western societies. In both animals and humans, the contributions of de novo cholesterol synthesis to biliary cholesterol secretion, as well as bile salt synthesis, are now considered to be of minor quantitative importance, with estimates as low as $10 \% .^{2}$ Clearly, the evidence suggests that biliary cholesterol and bile salt molecules derive principally from preformed cholesterol in plasma lipoproteins. ${ }^{2-4}$ Now, Hillebrant and colleagues (see page 700 ) have narrowed the focus and carried out a difficult and masterful study of patients with cholesterol gallstones with intentional total biliary diversions for a week following cholecystectomy, thereby maximising de novo bile salt synthesis. ${ }^{15}$ Short term extracorporeal apheresis of apolipoprotein B-100 containing lipoproteins (low density lipoprotein (LDL) and very low density lipoprotein (VLDL)) decreased plasma LDL cholesterol notably $(26 \%)$, and in parallel, the secretion rates of bile salts were also decreased significantly (31\%). Because of lipid coupling, the fall in bile salt secretion diminished the output of biliary phospholipid, as expected. ${ }^{1}$ Most noteworthy is that the plasma concentration of the lipoprotein particles devoid of apolipoprotein B-100, namely, plasma high density lipoprotein (HDL), did not change and biliary secretion rates of cholesterol remained unaffected.

The most parsimonious deduction from these observations is that a significant fraction of preformed cholesterol destined for bile salt synthesis is derived from plasma LDL/VLDL, which are taken up (the latter principally as IDL) by the liver by receptor mediated endocytosis. ${ }^{5}$ Hillebrant et al have unmasked this channel of cholesterol disposition by ensuring in their experimental design as much as a 10 -fold augmentation of bile salt synthesis. ${ }^{1}$ Presumably, following hydrolysis of the cholesteryl esters, which are in the hydrophobic cores of the apolipoprotein B-100 containing lipoproteins, cholesterol molecules move to sites of enzymes that initiate bile salt synthesis - cholesterol
$7 \alpha$-hydroxylase in the endoplasmic reticulum (the "neutral" pathway), and sterol 27-hydroxylase in mitochondria (the "acidic" pathway). ${ }^{1}$ The constant ratio (5:1) of the two primary bile salts in Hillebrant et al's work reflects the unwavering activity of both pathways during and after depletion of the LDL/VLDL cholesterol concentrations.

Epidemiological and clinical studies emphasise a significant negative correlation between the concentration of HDL cholesterol and atherosclerotic cardiovascular disease. ${ }^{5}$ Cholesterol efflux from cells is dependent principally on apolipoprotein AI, a protein with remarkable detergency and solubilising power, which forms nascent HDL particles by microsolubilisation of lipids from cellular membranes. ${ }^{7}$ The cholesterol holding capacity of these plasma "micelles" is greatly promoted by the activity of LCAT (lecithin:cholesterol acyltransferase), which is activated by apolipoprotein AI, esterifying the surface free cholesterol molecules of HDL with a fatty acid of the lecithin molecules. ${ }^{6}$ Once formed, cholesteryl esters diffuse to the hydrophobic cores of HDL and change the particle's shape from bilayer to ellipsoid. ${ }^{6}$ This allows the cholesterol deficient monomolecular layer on the particle's surface to continue its sterol salvage operation in peripheral tissues. The turnover of cholesteryl esters is accomplished in two ways: HDL transfers cholesteryl esters via a plasma cholesteryl ester transfer protein to the hydrophobic cores of LDL and $\mathrm{VLDL}^{6}{ }^{7}$; in addition, HDL transfers its free cholesterol and cholesteryl esters to hepatic parenchymal cells without the particle itself being engulfed. ${ }^{8}$ This contrasts with the receptor mediated endocytosis of other lipoproteins by hepatocytes. ${ }^{5}$

For over 20 years, evidence has accrued to suggest strongly that the unesterified cholesterol of HDL was preferentially utilised for biliary cholesterol secretion. This is the case in mammals irrespective of whether cholesterol is carried principally in HDL (rats), or in LDL (humans). ${ }^{2-4}$ The most logical deduction from Hillebrant et al's data is that free cholesterol of plasma HDL is directly channelled to biliary cholesterol secretion than to bile salt synthesis. Rates of plant sterol secretion into rat bile from lipoproteins of patients with hereditary phytosterolaemia support the secretory importance of HDL's unesterified cholesterol into bile cholesterol. ${ }^{9}$ Although cholesteryl esters in the core of HDL contribute to bile salt synthesis in rats, ${ }^{10}$ it is not known how much is destined for biliary secretion as free cholesterol. A calculation reveals that HDL's unesterified cholesterol could only be responsible for about $20 \%$ of the mass of cholesterol secreted into human bile daily, ${ }^{34}$ so based on Hillebrant et al's study, HDL's core lipid is a likely contributor. Appropriately, new insights spring from the powerful experimental methods molecular biology and molecular genetics.

It now seems that the hepatic receptor for HDL is the scavenger SR-BI receptor, ${ }^{8}$ which acts as a "docking ensemble" binding apolipoprotein AI and holding HDL particles sufficiently long in the space of Disse to allow their cholesteryl esters to enter the hepatocytes by an unknown mechanism. The "empty" HDL particle then continues in the circulation and repeats the uptake of 
unesterified cholesterol molecules from peripheral tissues, and their subsequent esterification and delivery to the liver. ${ }^{7}$ This recycling process from non-hepatic tissues to liver is known as reverse cholesterol transport. ${ }^{6}$ Recently, using an adenovirus vector, the SR-BI receptor has been overexpressed in murine hepatocytes, ${ }^{11}$ and the physiological result was the virtual disappearance of plasma HDL, a doubling of biliary cholesterol content, but no change in biliary bile salt concentrations. Obviously, the precise physiological function of this receptor has to be probed by knocking out the mouse gene, which should produce profound hyposecretion of cholesterol, and then doing the rescue experiment by introducing the human SR-BI gene into the knockout animals. In light of this new knowledge, Hillebrant and colleagues' study comes into perspective, and it seems totally consistent with other work ${ }^{2}$ emphasising ". . . that HDL cholesterol is more related to the biliary secretion of cholesterol than that of bile acids." All of this presages that HDL concentrations should be related to high cholesterol saturation of bile and prevalence of gallstones-yet the literature suggests an inverse relation between HDL cholesterol, bile cholesterol content, and gallstones. ${ }^{12}{ }^{13}$ Nevertheless, seeing that the genetic era of cholesterol gallstone research has dawned, ${ }^{14}$ perhaps there is a Lith gene that controls the upregulation of the SR-BI receptor and lithogenic bile formation.

In summary, the Hillebrant et al's study supports the concepts that (a) cholesterol molecules carried in the major plasma lipoproteins are the immediate source of both biliary steroids ${ }^{15}$ and $(b)$ compartmentalisation of lipoprotein cholesterol occurs within the liver with the cholesterol secreted into bile being derived from a pool of cholesterol that is distinct from the one used for bile salt synthesis. ${ }^{349}$ The time seems ripe for unravelling details of these intracellular pools as well as the routes of transhepatic traffic of cholesterol from blood to bile.

These pathophysiological concepts support two well known clinical observations of interest to gastroenterologists: with administration of competitive inhibitors ("statins") of HMG-CoA reductase, the rate limiting enzyme in cholesterol biosynthesis, bile does not become appreciably desaturated simply because of the minor contribution of de novo cholesterol synthesis. As a consequence, cholesterol gallstone dissolution does not seem to occur with these agents. When endogenous bile salt synthesis is profoundly suppressed-for example, with the administration of chenodeoxycholic acid (chenodiol) to attempt oral dissolution of cholesterol gallstones, an appreciable rise in LDL cholesterol concentration ensues. The converse is also the case, in that when faecal bile salt loss is augmented by large doses of cholestyramine or other orally administered binding polymers, by ileal bypass, or by ileal Crohn's disease, plasma LDL concentrations fall profoundly.

Department of Medicine, Harvard Medical School

MARTIN C CAREY

and Harvard Digestive Diseases Center,

Division of Gastroenterology,

Brigham and Women's Hospital, 75 Francis Street,

Boston, MA 02115, USA

1 Carey MC, Duane WC. Enterohepatic circulation. In: Arias IM, Boyer JL, Fausto N. Jakoby WB, Schachter D, Shafritz DA, eds. The liver: biology and pathobiology. 3rd edn. New York: Raven Press, 1994: 719-67.

2 Botham KM, Bravo E. The role of lipoprotein cholesterol in biliary steroid secretion. Studies with in vivo experimental models. Prog Lipid Res 1995; 34: 71-97.

3 Schwartz CC, Berman M, Vlahcevic ZR, Swell L. Multicompartmental analysis of cholesterol metabolism in man: Quantitative kinetic evaluation of precursor sources and turnover of high density lipoprotein cholesterol esters. F Clin Invest 1982; 70: 863-76.

4 Schwartz CC, Zech LA, van den Broek JM, Cooper PS. Cholesterol kinetics in subjects with bile fistula: Positive relationship between size of the bile acid precursor pool and bile acid synthetic rate. F Clin Invest 1993; 91: acid precur.

5 Brown MS, Goldstein JL. A receptor-mediated pathway for cholesterol homeostasis. Science 1986; 232: 34-7.

6 Tall AR, Small DM. Body cholesterol removal: Role of plasma high-density lipoproteins. Adv Lipid Res 1980; 17: 1-51.

7 Johnson WJ, Phillips MC, Rothtlat GM. Lipoproteins and cellular cholesterol homeostasis. In: R Bittman, ed. Subcellular biochemistry. Vol 28. Cholesterol: its functions and metabolism in biology and medicine. New York: Plenum Press, 1997: 235-76.

8 Acton S, Rigotti A, Landschulz KT, Xu S, Hobbs HH, Krieger M. Identification of scavenger receptor SR-BI as a high density lipoprotein receptor. Science 1996; 271: 518-20.

9 Robins SJ, Fasulo JM. High density lipoproteins, but not other lipoproteins provide a vehicle for sterol transport to bile. $\mathcal{F}$ Clin Invest 1997; 99: 380-4.

10 Pieters MN, Schouten D, Bakkeren HF, Esbach B, Brouwer A, Knook DL, et al. Selective uptake of cholesteryl esters from apolipoprotein-E-free
high-density lipoprotein by rat parenchymal cells in vivo is efficiently coupled to bile acid synthesis. Biochem f 1991; 280: 359-65.

11 Kozarsky KF, Donahee MH, Rigotti A, Igbal SN, Edelman ER, Krieger M. Overexpression of the HDL receptor SR-BI alters plasma HDL and bile Overexpression of the HDL receptor SR-BI
cholesterol levels. Nature 1997; 382: 414-7.

12 Thornton JR, Heaton KW, Macfarlane DG. A relation between highdensity-lipoprotein, cholesterol and bile cholesterol saturation. BMF 1981; 283: $1352-4$.

13 Thijs C, Knipschild P, Brombacher P. Serum lipids and gallstones: A casecontrol study. Gastroenterology 1990; 99: 843-9.

14 Khanuja B, Cheah Y-C, Hunt M, Nishina PM, Wang DQ-H, Chen HW, et al. Lithl, a major gene affecting cholesterol gallstone formation among inbred strains of mice. Proc Natl Acad Sci USA 1995; 92: 7729-33.

15 Empen K, Lange K, Stange EF, Scheibner J. Newly synthesized cholesterol in human bile and plasma: Quantitation by mass isotopomer distribution analysis. Am F Physiol 1997; 272: G367-73. 